\title{
Voice-controlled virtual assistants for the older people with visual impairment
}

Eye (2018) 32, 53-54; doi:10.1038/eye.2017.165; published online 4 August 2017

Compared with the other age groups, the elderly population in England and Wales suffer from the highest incidence of visual impairment, with almost 18000 Certificates of Vision Impairment (CVI) registrations, which was $75 \%$ of the total figure in the over-65 age group between 20122013. ${ }^{1}$ Age-related macular degeneration (AMD) is the leading cause of visual impairment, with estimations suggesting a $2.4 \%$ prevalence of late AMD in the over- 50 age group, equivalent to 513000 cases in the UK. The proportion of late AMD increases with advancing age, rising to 4.8 and $12.2 \%$ at over- 65 and over- 80 age groups respectively. ${ }^{2}$ Older people with visual impairment suffer from more physical and mental health co-morbidities compared to those with normal vision. ${ }^{3}$ This vulnerable group of population is also associated with higher rates of hospital admissions with falls. ${ }^{4}$ Psychologically, visually impaired elderly display higher prevalence of anxiety, depression, and have poorer quality of life. 5,6

It has been shown that introducing the Internet to the elderly can help to promote psychological well-being, contributing to their sense of empowerment and independence, through accessing news, weather and entertainment, obtaining information on interests and health problems, or shopping online. ${ }^{7,8}$ While high proportions of the working-age adults regularly surf the Internet, the older generation are less likely to do so. A recent survey in US found that Internet use among the elderly has steadily climbed over the past decade, with the 'younger seniors' (65-69year-olds) almost twice as likely to use the Internet than the over-80s, at 82 and $44 \%$ respectively. Not surprisingly, however, Internet use is also positively correlated to higher household income and educational attainment. ${ }^{9}$
'Virtual Assistants' are software agents powered by artificial intelligence, the first one popularised and used globally being Apple Inc.'s Siri. ${ }^{10}$ It was integrated into the iOS (iPhone operating system) and was first released in 2011. To date other notable Virtual Assistants, backed by competing tech giants, include Amazon Inc.'s Alexa, Google Inc.'s Assistant, and Microsoft Corp's Cortana.

'Traditional' devices such as computers, smartphones, and tablets rely on display screens to present information and keyboards or touchscreens to input data or commands. These require reasonable levels of vision and manual dexterity, both of which can be demanding for older persons with degenerative eye and joint diseases. In contrast, voice-driven 'smart speakers' powered by Virtual Assistants are able to bypass these constraints, relying on the users' speech and hearing functions instead.

Amazon Echo, publicly launched in 2016, is the first always-on voice-controlled smart speaker powered by 'Alexa'. The use of such a gadget can assist the day-to-day living of an elderly person with visual impairment. With Internet connection, its users can listen to the news, music, radio channels and audio books. They can also check the time, set timers and alarms, organise personal calendars, search for facts and shop online, all via voice commands alone. With other 'smart' home devices installed, one can control the thermostat and lights, all without the need of physical contact. ${ }^{11}$ For the first time, a person with reduced vision and no prior experience with computers can utilise such hi-tech gadgets simply by talking to them.

These valuable tools do face significant barriers to gaining traction in this elderly cohort; it is important to note that the very elderly and socially deprived suffer from a higher risk of vision-related quality of life impairment ${ }^{12}$ incidentally the very group of older people least likely to surf the Internet and possibly least able to afford such devices and home broadband 
connections. Furthermore, elderly with co-existing hearing or speech impairment, such as from cerebrovascular accidents, may not benefit from such devices. At the moment certain simple functions, for example deleting items off a shopping list on the Alexa, cannot be voice-controlled and is still display screendependent. There are also concerns regarding possible intrusion to privacy with these always-on microphoneenabled products that are connected to the Internet. ${ }^{13}$

Undoubtedly, we as healthcare professionals will encounter more and more tech-savvy elderly patients in the years to come. Will these voice-controlled smart speakers assist the daily living of older people with visual impairment and enrich their psychological well-being? Perhaps; more real-world studies will certainly be needed to evaluate their efficacies. Optimising costs and functionality to make them more accessible and userfriendly to this population will be a necessary consideration for technology firms, healthcare services and patient support groups alike, in order to reap the full benefit this technology can bring.

\section{Conflict of interest}

The author declares no conflict of interest.

\section{Acknowledgements}

The author would like to thank Mrs Betty Reynolds for her insightful contributions from the patient's perspective.

\section{References}

1 Quartilho A, Simkiss P, Zekite A, Xing W, Wormald R, Bunce C. Leading causes of certifiable visual loss in England and Wales during the year ending 31 March 2013. Eye 2016; 30(4): 602-607.

2 Owen CG, Jarrar Z, Wormald R, Cook DG, Fletcher AE, Rudnicka AR. The estimated prevalence and incidence of late stage age related macular degeneration in the UK. $\mathrm{Br} J$ Ophthalmol 2012; 96(5): 752-756.

3 Court H, McLean G, Guthrie B, Mercer SW, Smith DJ. Visual impairment is associated with physical and mental comorbidities in older adults: a cross-sectional study. BMC Med 2014; 12: 181.

4 Jack CI, Smith T, Neoh C, Lye M, McGalliard JN. Prevalence of low vision in elderly patients admitted to an acute geriatric unit in Liverpool: elderly people who fall are more likely to have low vision. Gerontology 1995; 41(5): 280-285.

5 Li Y, Crews JE, Elam-Evans LD, Fan AZ, Zhang X, Elliott AF et al. Visual impairment and health-related quality of life among elderly adults with age-related eye diseases. Qual Life Res 2011; 20(6): 845-852.

6 van der Aa HP, Comijs HC, Penninx BW, van Rens GH, van Nispen RM. Major depressive and anxiety disorders in visually impaired older adults. Invest Ophthalmol Vis Sci 2015; 56(2): 849-854.

7 Shapira N, Barak A, Gal I. Promoting older adults' wellbeing through Internet training and use. Aging Ment Health 2007; 11(5): 477-484.

8 Mellor D, Firth L, Moore K. Can the Internet improve the well-being of the elderly? Ageing International 2008; 32 (1): $25-42$.

9 Anderson M, Perrin A. Tech Adoption Climbs Among Older Adults. Pew Internet \& American Life Project: Washington, DC, 2017. Retrieved 3 June 2017.

10 Apple Inc. iOS-Siri 2017. Available at http://www.apple. $\mathrm{com} / \mathrm{ios} / \mathrm{siri} /$.

11 RNIB. How does the Amazon Echo help people with a vision impairment?; 2017. Available at http://www.rnib.org.uk/ nb-online/how-does-amazon-echo-help-disabled-people.

12 Frost A, Eachus J, Sparrow J, Peters TJ, Hopper C, Davey-Smith G et al. Vision-related quality of life impairment in an elderly UK population: associations with age, sex, social class and material deprivation. Eye 2001; 15 (Pt 6): 739-744.

13 Gray S. Always on: privacy implications of microphoneenabled devices: Federal Trade Commission; 2016. Available at https:/ /www.ftc.gov/system/files/documents/public_ comments/2016/08/00003-128652.pdf.

D K-h Ho

Department of Ophthalmology, Royal Gwent Hospital, Newport, UK

Correspondence: D K-h Ho, Department of Ophthalmology, Royal Gwent Hospital, Cardiff Rd, Newport NP20 2UB, UK Tel: +44 (0)1633 234 234; Fax: +44 (0)1633 656294 . E-mail: derek.ho@doctors.org.uk 